\title{
Medical Image of the Week: Finger in Glove
}

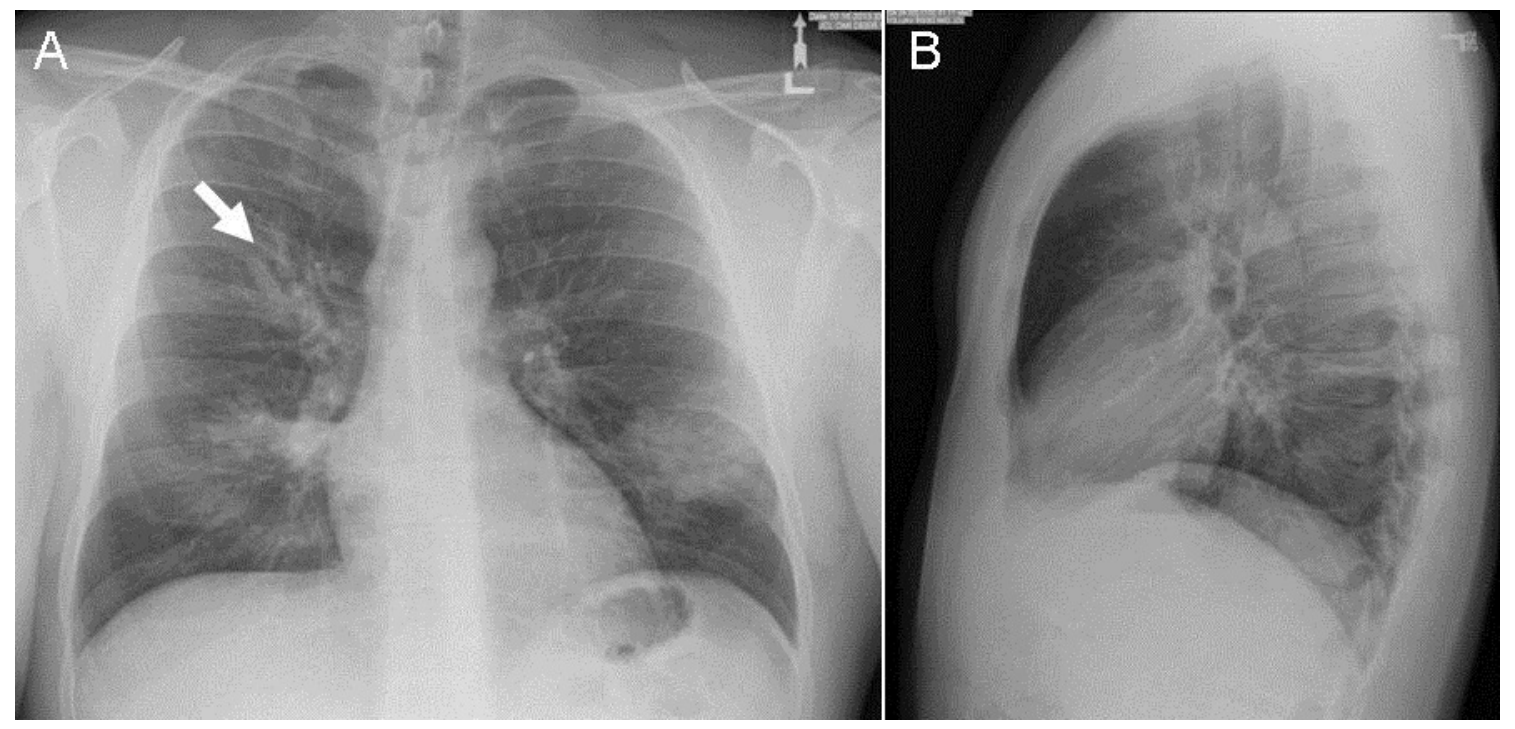

Figure 1. PA (Panel A) and lateral (Panel B) chest $x$-ray showing finger in glove (arrow) in the right upper lung with other scattered areas of consolidation.
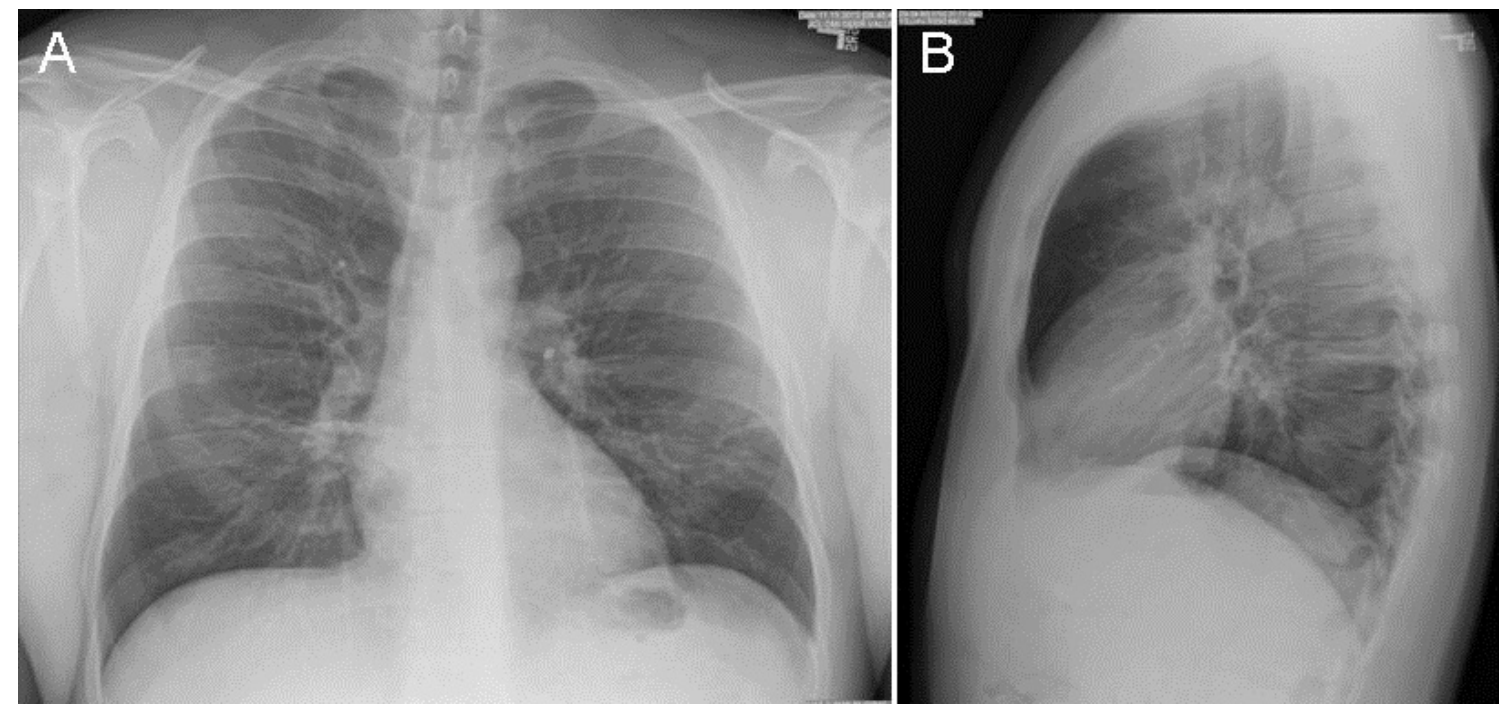

Figure 2. Repeat chest x-ray about a month later showing generalized clearing.

A 45-year old man with a history of asthma presented with increasing shortness of breath, and cough productive of dark firm plugs, sometimes branching. His chest X-ray (Figure 1) shows finger in glove (arrow) in the right upper lung with other scattered areas of consolidation. His serum IgE was elevated at $750 \mathrm{IU} / \mathrm{ml}$ (normal $<380 \mathrm{IU} / \mathrm{ml}$ ). His eosinophil count was $12 \%$. Aspergillus IgE was 6.69 (normal< 0.35 ). A diagnosis of probable allergic bronchopulmonary aspergillosis 
(ABPA) was made. He was given oral corticosteroids. Follow up about a month later showed dramatic clinical improvement and a repeat chest x-ray (Figure 2) showed generalized clearing.

The initial chest x-ray shows a "finger in glove" pattern in the right upper lobe (Figure 1, arrow), which is due to mucoid impaction within the larger bronchi (1). The same appearance has also been referred to as the rabbit ear appearance, Mickey Mouse appearance, toothpaste-shaped opacities, Y-shaped opacities, Vshaped opacities and the Churchill sign because it resembles the " $\mathrm{V}$ " gesture often associated with Winston Churchill.

ABPA is an immunological pulmonary disorder caused by hypersensitivity to Aspergillus fumigatus, manifesting with poorly controlled asthma, recurrent pulmonary infiltrates and central bronchiectasis (2). Primary therapy consists of oral corticosteroids to control exacerbations, itraconazole as a steroid-sparing agent and optimized asthma therapy. Uncertainties surround the prevention and management of bronchiectasis, chronic pulmonary aspergillosis and aspergilloma as complications, concurrent rhinosinusitis, environmental control and long-term management.

Gerald F. Schwartzberg, MD

Phoenix, AZ

\section{References}

1. Weerakkody $\mathrm{Y}$, Jones J. Finger in glove sign. Available at: http://radiopaedia.org/articles/finger-in-glove-sign (accessed 11/22/13).

2. Agarwal R, Chakrabarti A, Shah A, Gupta D, Meis JF, Guleria R, Moss R, Denning DW; ABPA complicating asthma ISHAM working group. Allergic bronchopulmonary aspergillosis: review of literature and proposal of new diagnostic and classification criteria. Clin Exp Allergy. 2013;43(8):850-73. [CrossRef] [PubMed] 